ARTIGO DE REVISÃO REVIEW ARTICLE

Palavras-chave:

análise de impacto orçamentário, avaliação de tecnologias em saúde, gestão de saúde

\section{Análise de impacto orçamentário: uma revisão prática de conceitos e aplicações para o gestor}

\author{
Budget impact analysis: a practical review of \\ concepts and applications for the manager
}

Miguel Francisco Bezerra de Medeiros', Lucas Miyake Okumura', Bruno Salgado Riveros ${ }^{1,2}$, Rosa Camila Lucchetta1,2, Mariana Rosim ${ }^{1,3}$, Marcelo Eidi Nita ${ }^{1,4}$

DOI: 10.21115/JBES.v10.n1.p75-9

\section{RESUMO}

Avaliações econômicas em saúde são essenciais para a tomada de decisão de gestores, visto que as inovações no setor nem sempre podem ser incorporadas conforme as expectativas dos usuários e da indústria. Logo, a análise de impacto orçamentário (AIO), uma das principais ferramentas da avaliação de tecnologias em saúde (ATS), permite aos gestores estimar o potencial número de indivíduos elegíveis para determinada tecnologia ao longo dos anos, prever quanto será necessário gastar para incorporar a tecnologia ou, eventualmente, se haverá economia nos cofres do pagador com a inclusão dela. Com a crescente publicação de artigos científicos sobre AIO no Brasil, surge a preocupação a respeito da qualidade metodológica desses estudos. O objetivo desta revisão é apresentar recomendações-chave para a elaboração de uma AlO adequada e expor a utilidade prática da AIO para a tomada de decisão de gestores de saúde por meio da análise de quatro estudos publicados. O formato de apresentação de uma AIO possibilita rápido entendimento e possui atributos que permitem evidenciar o maior valor de produtos e serviços de saúde junto aos tomadores de decisão em saúde, contribuindo para as melhores escolhas do ponto de vista clínico e econômico, nos sistemas público e privado. Porém, cabe ressaltar que alguns estudos ainda carecem de evidências de mundo real ou dados epidemiológicos para as estimativas e trabalham de maneira insuficiente as ferramentas para a redução de incertezas paramétricas.

\section{Keywords:}

budget impact analysis, health technology assessment, health management

\begin{abstract}
Health economics evaluations are essential for decision makers, since innovations can not always be incorporated according to expectations of users and industry. Therefore, the Budget Impact Analysis (BIA), one of the main tools in Health Technology Assessment (HTA), allows managers to estimate the potential number of individuals eligible for a given technology over the years, to predict how much it will be necessary to spend for incorporate the technology or, eventually, whether there will be savings in the payer's coffers with the inclusion of the same. With the growing publication of scientific articles on BIA in Brazil, there is concern about the methodological quality of these studies. The objective of this review is to present key recommendations to elaborate an adequate BIA, commenting on four published studies, aiming to expose the practical utility of BIA for health managers. The presentation format of the BIA provides a quick understanding and has attributes that allows evidence of the highest value of health products and services among health decision makers, contributing to the best clinical and economic choices in public and private health systems. However, it should be noted in some studies there is still a lack of real-world evidence or epidemiological data for the estimates and insufficient using of tools to reduce parametric uncertainties.
\end{abstract}

Recebido em: 18/02/2018. Aprovado para publicação em: 26/03/2018.

1. MAPESolutions, São Paulo, SP, Brasil.

2. Universidade Federal do Paraná (UFPR), Curitiba, PR, Brasil.

3. Universidade de São Paulo (USP), SP, São Paulo, Brasil.

4. Hospital Alemão Oswaldo Cruz, SP, São Paulo, Brasil.

Instituição onde o trabalho foi executado: MAPESolutions.

Autor correspondente: Bruno S. Riveros. Av. Fagundes Filho, 191, sala 58, São Judas, São Paulo, SP. CEP: 04304-000.

Telefone: (041) 99192-4833. E-mail: bruno.riveros@mapesolutions.com 


\section{Introdução}

A saúde é um dos setores da sociedade que mais gera inovação em serviços e produtos, acarretando gastos, os quais nem sempre compensam sua incorporação a médio e longo prazo. Nesse contexto, a análise de impacto orçamentário (AIO), uma das principais ferramentas da avaliação de tecnologias em saúde (ATS), permite aos gestores estimar o potencial número de indivíduos elegíveis e gastos projetados para determinada tecnologia ao longo dos anos. Além disso, é capaz de prever quanto será necessário investir para incorporar a tecnologia ou, eventualmente, se haverá redução de custos nos cofres do pagador com a inclusão dela (Ministério da Saúde (Brasil). Secretaria de Ciência Tecnologia e Insumos Estratégicos. Departamento de Ciência e Tecnologia, 2012; Nita et al., 2009).

Diversas agências de ATS e órgãos públicos e privados em saúde dependem desses estudos para decidir pela incorporação ou exclusão de tecnologias nos sistemas de saúde. Alguns exemplos são o Canadian Agency for Drugs and Technologies in Health (CADTH - Canadá) e o Pharmaceutical Benefits Advisory Committee (PBAC - Austrália). Mesmo no Reino Unido, uma nação onde os aspectos de custo-efetividade dominam a tomada de decisão, a AIO vem ganhando mais espaço e relevância, pela capacidade de projeção orçamentária ao longo do tempo. No contexto brasileiro, a Comissão Nacional de Incorporação de Tecnologias no Sistema Único de Saúde (Conitec) e o Comitê Permanente de Regulação da Atenção à Saúde (Cosaúde) são as entidades que avaliam dossiês que requerem, entre outros estudos, a AlO para avaliar uma tecnologia em saúde. Mais recentemente, comitês representativos de operadoras de saúde (OPS) têm se mobilizado para avaliar novas tecnologias ainda não incorporadas no rol da Agência Nacional de Saúde Suplementar (ANS). Essa iniciativa ocorre justamente pela compreensão das OPS de que algumas tecnologias são verdadeiras oportunidades, não somente para aprimorar o cuidado à saúde, mas também para amenizar as pressões diversas em seus orçamentos.

É notável a crescente publicação de artigos científicos sobre AIO no Brasil. Paralelo a esse crescimento, surge a preocupação a respeito da qualidade metodológica desses estudos que estão sendo publicados. Diante das metodologias empregadas nos estudos de AIO identificados no Brasil, este artigo de revisão apresenta recomendações-chave para a elaboração de uma AlO adequada do ponto de vista metodológico. Ainda, este artigo comenta quatro estudos publicados e expõe a utilidade prática da AIO para a tomada de decisão de gestores de saúde, bem como traça uma visão crítica sobre eles.

\section{Definição de análise de impacto orçamentário}

Para fins de definição e análise crítica das AIOs publicadas no Brasil, foram utilizados os seguintes materiais: 1) Princípios de boas práticas para elaboração de $\mathrm{AlO}$, elaborados pela International Society for Pharmacoeconomics and Outcomes Research (ISPOR) (Sullivan et al., 2014); 2) Diretrizes Metodológicas - análise de impacto orçamentário: manual para o Sistema de Saúde do Brasil, elaborado pelo Ministério de Saúde (MS) (Ministério da Saúde (Brasil). Secretaria de Ciência Tecnologia e Insumos Estratégicos. Departamento de Ciência e Tecnologia, 2012). Tais referências serviram como arcabouço teórico da estrutura de uma AlO e foram traduzidas em uma tabela como uma lista de itens imprescindíveis para esse tipo de estudo, sob a perspectiva do gestor ou do tomador de decisão.

A Tabela 1 serviu como base para a análise e interpretação de quatro estudos, incluindo diferentes tecnologias em

Tabela 1. Itens imprescindíveis em uma análise de impacto orçamentário e sua aplicabilidade ao gestor

\begin{tabular}{|c|c|}
\hline Itens da AIO & Aplicabilidade ao gestor \\
\hline Perspectiva & $\begin{array}{l}\text { Designar quem detém o orçamento } \\
\text { e decidirá por pagar ou não pela } \\
\text { tecnologia em determinado sistema de } \\
\text { saúde, de acordo com a realidade. }\end{array}$ \\
\hline Modelo analítico & $\begin{array}{l}\text { Reconhecer que as etapas da história } \\
\text { natural da doença estão bem descritas } \\
\text { e, assim, fornecem estimativas } \\
\text { adequadas para as etapas seguintes } \\
\text { da análise de impacto orçamentário. }\end{array}$ \\
\hline População-alvo & $\begin{array}{l}\text { Delimitar a população elegível possibilita } \\
\text { ao gestor analisar se a alocação de } \\
\text { recursos é prioritária, em detrimento } \\
\text { do restante da população. }\end{array}$ \\
\hline $\begin{array}{l}\text { Cenário de } \\
\text { referência }\end{array}$ & $\begin{array}{l}\text { Saber como o sistema de saúde trata } \\
\text { essas pessoas atualmente e quais são } \\
\text { os custos do tratamento atual. }\end{array}$ \\
\hline Cenário alternativo & $\begin{array}{l}\text { Ter informações sobre a alternativa } \\
\text { sugerida, enxergar cenários alternativos } \\
\text { e comparar com o atual. }\end{array}$ \\
\hline $\begin{array}{l}\text { Difusão da } \\
\text { tecnologia }\end{array}$ & $\begin{array}{l}\text { Saber se as propostas de velocidade } \\
\text { de incorporação e proporções de } \\
\text { adoção para a tecnologia são viáveis, } \\
\text { de acordo com o orçamento. }\end{array}$ \\
\hline Fonte de dados & $\begin{array}{l}\text { Garantir que a origem dos dados } \\
\text { apresentados seja confiável. }\end{array}$ \\
\hline $\begin{array}{l}\text { Horizonte } \\
\text { temporal }\end{array}$ & $\begin{array}{l}\text { Auxiliar no planejamento para alocação } \\
\text { de recursos ao longo de tempo. }\end{array}$ \\
\hline $\begin{array}{l}\text { Análise de } \\
\text { sensibilidade }\end{array}$ & $\begin{array}{l}\text { Simular cenários com alterações em } \\
\text { custos, população de interesse ou fatias de } \\
\text { mercado permite prever se a incorporação } \\
\text { de tecnologia é viável diante de incertezas } \\
\text { no futuro. Também reduz as incertezas } \\
\text { sobre as estimativas obtidas na análise. }\end{array}$ \\
\hline Validação & $\begin{array}{l}\text { Aumentar a confiança nas estimativas } \\
\text { obtidas e consequentemente } \\
\text { fornecer maior suporte técnico } \\
\text { para a decisão do gestor. }\end{array}$ \\
\hline
\end{tabular}

Adaptada de: Sullivan et al. (2014); Ministério da Saúde (Brasil), 2012. 
saúde (Entringer et al., 2013; Nita et al., 2012; Peixoto et al., 2017; Senna et al., 2013), como material médico hospitalar, teste diagnóstico, implementação de cuidados e medicamentos para doenças adquiridas ao longo da vida.

\section{AlO para material médico hospitalar: oclusor percutâneo septal para o Sistema Único de Saúde (SUS)}

Senna et al. (2013) realizaram AIO da incorporação de oclusor septal percutâneo para o tratamento de defeitos do septo atrial do tipo ostium secundum (OS), sob a perspectiva do SUS, em um horizonte temporal de cinco anos (Senna et al., 2013). O cenário de referência foi a cirurgia torácica, principal tratamento na época. Os autores determinaram a população elegível de acordo com dados epidemiológicos e critérios clínicos de elegibilidade para implante do oclusor descritos em literatura, conforme a Tabela 2.

Os métodos para estimar o número de pessoas elegíveis para a tecnologia em questão e o modelo analítico são o cerne de uma AIO. No caso do artigo de Senna et al. (2013), os autores obtiveram o impacto orçamentário por meio de um modelo estático, multiplicando o número de pacientes pelos respectivos custos (oclusor ou cirurgia), e posteriormente simularam cenários com as proporções de cada tratamento para o grupo elegível. Esse é o método mais simples para chegar ao resultado do impacto orçamentário incremental.

Cabe destacar que os autores executaram análise de sensibilidade probabilística de Monte Carlo para simular outras possibilidades, como variações nos preços do oclusor e nas proporções de adoção das tecnologias em questão (oclusor e cirurgia torácica). No entanto, não foi realizada a validação desse modelo.

\section{AlO para diagnóstico: o caso da malária na Amazônia brasileira}

A AIO é parte de uma ATS, em que podem ser avaliados medicamentos, procedimentos médicos, equipamentos e, até mesmo, programas de cuidados para saúde (Nita et al., 2009).
Peixoto et al. (2017), por exemplo, analisaram um método rápido para diagnóstico da deficiência de G6PD (glicose-6-fosfato desidrogenase), problema que pode desencadear anemia hemolítica em pacientes do sexo masculino com malária por Plasmodium vivax e sob tratamento com quininas, no contexto do SUS, problema relevante na região da Amazônia brasileira. Nesse caso não havia método específico para esse diagnóstico no SUS. Dessa forma, os autores propuseram os cuidados atuais como referência (diagnóstico e tratamento de malária) e horizonte temporal de três anos. Com base em opiniões de especialistas, foram adotadas as respectivas proporções crescentes de uso da tecnologia no contexto do SUS: 30\%, 70\% e 100\%.

Devido à complexidade dos possíveis estados de saúde ao longo do tempo, os autores usaram o modelo analítico de Markov para estimar coortes hipotéticas de pacientes, levando em conta a incidência de malária em anos anteriores, estimativas das incidências para os anos seguintes e estimativas da subpopulação com deficiência de G6PD. Posteriormente, foram estimadas as probabilidades de transições para outros estados de saúde e eventos ao longo de 12 meses (por exemplo, tratamento medicamentoso, hospitalizações, recidiva, cura ou morte).

Os custos das tecnologias, procedimentos e eventos foram obtidos de estudos prévios. Com exceção do custo da consulta especializada, a análise de sensibilidade escolhida pelos autores foi a determinística univariada e considerou custos (de custo de exames laboratoriais à assistência médico-hospitalar dos pacientes que falham à terapia) e probabilidades relevantes para o escopo do estudo (de deficiência de G6PD à proporção de pacientes que falham com quinina). Os autores chegaram à conclusão de que nenhuma das variáveis supracitadas foi capaz de alterar as estimativas finais de impacto orçamentário, respectivamente do ano 1 ao ano 3: -34.601,30 USD, -64.480,53 USD e -73.729,26 USD. Apesar do impacto positivo (a tecnologia traz redução de custos ao longo dos anos), este estudo também não realizou validação do modelo.

Tabela 2. Número estimado de pacientes com defeitos do septo atrial do tipo ostium secundum (OS) - Brasil, 2010-2015

\begin{tabular}{lccccc}
\hline Período & Nascidos vivos & $\begin{array}{c}\text { Cardiopatias } \\
\text { congênitas } \\
\mathbf{( 9 / 1 . 0 0 0 )}\end{array}$ & $\begin{array}{c}\text { Defeitos do } \\
\text { septo atrial } \\
(\mathbf{1 0 \% )}\end{array}$ & $\begin{array}{c}\text { Tipo OS (75\%) } \\
\text { Proporção de } \\
\text { pacientes elegíveis } \\
\text { ao implante do } \\
\text { oclusor (75\%) }\end{array}$ \\
\hline $\mathbf{2 0 1 0}$ & 2.861 .868 & 25.757 & 2.576 & 1.932 & 1.449 \\
\hline $\mathbf{2 0 1 1}$ & 2.846 .731 & 25.621 & 2.562 & 1.932 & 1.442 \\
\hline $\mathbf{2 0 1 2}$ & 2.835 .108 & 25.516 & 2.552 & 1.914 & 1.436 \\
\hline $\mathbf{2 0 1 3}$ & 2.826 .183 & 25.436 & 2.544 & 1.908 & 1.431 \\
\hline $\mathbf{2 0 1 4}$ & 2.819 .330 & 25.374 & 2.537 & 1.903 & 1.427 \\
\hline $\mathbf{2 0 1 5}$ & 2.814 .067 & 25.327 & 2.533 & 1.900 & 1.425 \\
\hline
\end{tabular}

Nascidos vivos em 2010: dados oficiais; nascidos vivos entre 2011 e 2015, estimativas; Adaptada de: Senna et al. (2013). 


\section{AlO para implementação de serviços: o método Canguru}

Entringer et al. (2013) usaram um modelo de decisão analítico (árvore de decisão) para simular os custos do método Canguru e de unidade intermediária neonatal no Rio de Janeiro, sob perspectiva do SUS.

A população-alvo foi constituída pelos recém-nascidos estáveis clinicamente, elegíveis para receber assistência nas duas modalidades de cuidado supracitados. O impacto orçamentário foi estimado para o horizonte temporal de um ano, e a proporção de recém-nascidos elegíveis para as duas modalidades foi obtida por coleta de dados nas maternidades incluídas no estudo. As probabilidades dos eventos e os custos no período da assistência foram incorporados ao modelo.

Foi desenvolvida a análise de sensibilidade determinística por cenário, em que as taxas de adoção do método Canguru foram variadas em maior ou menor escala, para comparação com o cenário de referência (cuidado usual em unidade neonatal). Apesar do modelo também não ter sido validado, observou-se que há crescente redução de gastos quando há maiores proporções de pacientes atendidos pelo método Canguru, chegando a -380 mil reais quando 50\% dos neonatos recebem o método e -1 milhão de reais se as taxas chegarem a 100\%. Do contrário, há gasto incremental de 300 mil reais em um cenário com 100\% de pacientes atendidos pelo método usual.

\section{AIO para saxagliptina como terapia adicional à metformina}

Nita et al. (2012) usaram modelo analítico de simulação de eventos discretos para comparar três terapias adicionais à metformina (saxagliptina, pioglitazona e rosiglitazona) em pacientes com diabetes mellitus tipo 2 sem controle adequado da glicemia.

Esses autores consideraram um impacto orçamentário da incorporação desses medicamentos num período de três anos, sob a perspectiva de uma OPS. As incertezas paramétricas foram submetidas à análise de sensibilidade determinística univariada, considerando as variáveis de prevalência de diabetes, participação do produto no mercado e custos de aquisição do medicamento. Não houve citação quanto à validação do modelo.

Os principais resultados do estudo demonstraram que o uso de saxagliptina foi dominante ao ser comparado com os fármacos da classe das glitazonas. Em termos de $\mathrm{AlO}$, relativamente ao custo de aquisição de 2 mil reais por paciente, verificou-se que em três anos haveria o reembolso dessa tecnologia ao pagador, por meio de redução de gastos projetados em 417 mil reais (6,6\% no terceiro ano).

\section{Oportunidades e fragilidades}

Entre os estudos destacados, a maioria fez alusão aos itens que são imprescindíveis para elaborar uma AIO (Tabela 1).
Contudo, salientamos a relevância de outras etapas metodológicas a serem cumpridas no desenvolvimento de uma AlO, visando à solidez e à utilidade prática.

Idealmente, a AlO é precedida de outras etapas, nas quais é estabelecida a segurança, eficácia e efetividade de determinada tecnologia. Dessa forma, a tarefa da indústria, gestores, sociedades médicas e provedores de serviços de saúde é apropriar-se desse modelo para evidenciar o valor do seu produto ou serviço aos tomadores de decisão em saúde e oferecer as melhores escolhas do ponto de vista não só clínico, mas também econômico, visando ao uso mais eficiente de recursos dos sistemas de saúde.

No SUS, a Sociedade Brasileira de Hemodinâmica e Cardiologia Intervencionista demandou a incorporação de oclusores para fechamento percutâneo em pacientes com comunicação interatrial. A recomendação da Conitec foi favorável à incorporação no SUS como alternativa à cirurgia tradicional, beneficiando mais de 1.000 pacientes ao ano com o uso da nova tecnologia e potencialmente gerando economia da ordem de R\$ 7 milhões para o SUS, além de benefícios intangíveis (sem necessidade de esternotomia, circulação extracorpórea ou internação em unidade de terapia intensiva). O estudo de Senna et al. (2013), anteriormente citado, trata justamente de AIO relacionada à demanda avaliada pela Conitec (Conitec, 2014).

Outro atributo da AlO é que geralmente os resultados de uma AIO são apresentadas em um formato que possibilitam rápido entendimento, independentemente do grau de familiaridade com o tema e/ou área de formação do tomador de decisão. Porém, há de se registrar que os resultados do impacto orçamentário são estimativas, compreendidas numa gama de probabilidades e que apresentam graus de incerteza. Assim, análises de sensibilidades são essenciais para estimar o impacto dessas incertezas paramétricas, sendo comum observar predileção por análises determinísticas univariadas, que consistem em alterar uma variável de cada vez, em detrimento das análises probabilísticas, as quais levam em conta a variância (e eventualmente a covariância) dos parâmetros utilizados no modelo matemático.

Nesse sentido, é necessário rigor metodológico, desde a busca e a obtenção de dados altamente confiáveis (por exemplo, uso de dados de vida real representativos e parâmetros de difusão da tecnologia ou market share - proporção da tecnologia no mercado - bem fundamentados), cálculo epidemiológico da população-alvo até a utilização das análises de sensibilidade e validação que consigam explorar ao máximo a variabilidade, confiabilidade e incertezas, com o intuito de simular cenários que se aproximem o máximo possível do mundo real.

Nesse sentido, Faleiros et al. (2016) desenvolveram revisão sistemática sobre AIO de medicamentos e identificaram 92 artigos publicados entre 2001 e 2015, entre os quais 95\% eram provenientes dos EUA ou da Europa, com o objetivo de verificar se esses trabalhos cumpriam os itens preconizados 
pela ISPOR. Nenhum dos artigos cumpriu os nove itens do checklist; apenas 24\% deles expuseram claramente que os métodos para a derivação de estimativas se baseavam em alguma diretriz metodológica; 27\% não apresentaram análise de sensibilidade e apenas $5 \%$ desses artigos reportaram testes de validação do modelo.

\section{Considerações finais}

Este artigo visa aproximar o leitor dos conceitos da AIO por meio da contextualização em algumas publicações, sem pretensão de esgotar o assunto ou de se colocar como marco teórico. A produção deste material inclusive revela a necessidade de uso mais amplo da ferramenta com o intuito e o desafio de proporcionar as melhores intervenções que aliem aspectos clínicos, econômicos e éticos.

Por fim, este artigo busca construir alicerces para maior produção científica desse tipo no Brasil, bem como uma exploração mais densa e crítica, por exemplo, por meio de revisão sistemática de literatura acerca do tema.

\section{Referências bibliográficas}

Entringer AP, Pinto MT, Magluta C, Gomes MASM. Impacto orçamentário da utilização do Método Canguru no cuidado neonatal. Rev Saúde Pública. 2013;47(5):976-83.

Faleiros DR, Álvares J, Almeida AM, de Araújo VE, Andrade El, Godman BB, et al. Budget impact analysis of medicines: updated systematic review and implications. Expert Rev Pharmacoecon Outcomes Res. 2016;16(2):257-66.
Ministério da Saúde (Brasil). Secretaria de Ciência Tecnologia e Insumos Estratégicos. Departamento de Ciência e Tecnologia. Diretrizes Metodológicas: Análise de Impacto Orçamentário: Manual para o Sistema de Saúde do Brasil. Série A: Normas e Manuais Técnicos; 2012.

Ministério da Saúde (Brasil). Secretaria de Ciência, Tecnologia e Insumos Estratégicos.

Departamento de Gestão e Incorporação de Tecnologias em Saúde. Relatório de Recomendação da Comissão Nacional de Incorporação de Tecnologias no SUS -Conitec - 102: Fechamento de comunicação interatrial septal (CIA) por dispositivo percutâneo 2014; p. 59.

Nita ME, Campino A, Secoli S, Sarti F, Nobre M, Costa A, et al. Avaliação de Tecnologias em Saúde: evidência clínica, análise econômica e análise de decisão. Porto Alegre: Artmed; 2009.

Nita ME, Eliaschewitz FG, Ribeiro E, Asano E, Barbosa E, Takemoto M, et al. Custo-efetividade e impacto orçamentário da saxagliptina como terapia adicional à metformina para o tratamento do diabetes mellitus tipo 2 no sistema de saúde suplementar do Brasil. Rev Assoc Med Bras 2012:58(3):294-301.

Peixoto HM, Brito MA, Romero GA, Monteiro WM, de Lacerda MV, de Oliveira MR. Rapid diagnostic test for G6PD deficiency in Plasmodium vivaxinfected men: a budget impact analysis based in Brazilian Amazon. Trop Med Int Health. 2017;22(1):21-31.

Senna KMS, Costa MG, Tura BR, Correia MG, Santos MS. Análise de impacto orçamentário do oclusor septal percutâneo para o fechamento de comunicação interatrial (CIA) do tipo ostium secundum. Value Health. 2013;16(7):A704.

Sullivan SD, Mauskopf JA, Augustovski F, Jaime Caro J, Lee KM, Minchin M, et al. Budget impact analysis - principles of good practice: report of the ISPOR 2012 Budget Impact Analysis Good Practice II Task Force. Value Health. 2014;17(1):5-14. 